\title{
REVISIÓN
}

\section{La funcionalidad de los óxidos de hierro ${ }^{(\bullet)}$}

\author{
J.G. Castaño ${ }^{(*)}$ y C. Arroyave ${ }^{(*)}$
}

\begin{abstract}
Resumen Algunos óxidos de hierro tienen aplicaciones interesantes para las tecnologías actuales y futuras. Aparte de poseer propiedades adecuadas para cumplir diversas funciones, son relativamente abundantes en la naturaleza o su obtención en forma sintética no es complicada. En este artículo se presentan cinco de ellos (hematita, magnetita, maghemita, goethita y akaganeíta) y su utilización en campos como la industria química, la biotecnología, la medicina, los nuevos materiales y el electromagnetismo.
\end{abstract}

Palabras clave: Magnetita. Hematita. Maghemita. Goethita. Akaganeíta.

\section{Functionality of the iron oxides}

\begin{abstract}
Some iron oxides have a great scientific and technological possibilities, not only for their importance in the present, but also for their great potential in the development of the future technologies. They have adequate properties to carry out several functions. They are plentiful in the nature and their synthetic obtention is not complex. This paper shows five of them (hematite, magnetite, maghemite, goethite and akaganeite) and their utilization in fields like chemical industry, biotechnology, medicine, new materials and electromagnetism.
\end{abstract}

Keywords: Magnetite. Hematite. Maghemite. Goethite. Akaganeite.

\section{INTRODUCCIÓN}

Cuando se hace referencia a los materiales de interés tecnológico, se puede distinguir entre los denominados estructurales, destinados a soportar los diferentes tipos de cargas mecánicas y térmicas en estructuras, montajes, piezas de máquinas y equipos, y los materiales funcionales seleccionados para cumplir una función específica basada en sus propiedades magnéticas, ópticas, electrónicas o catalíticas. Entre estos últimos se hallan numerosos compuestos inorgánicos que incluyen los óxidos metálicos y, en particular, los óxidos de hierro como una familia de sustancias de amplias posibilidades de aplicación.

El hierro, como metal de transición que es, tiene la capacidad de formar diversos compuestos oxidados, algunos de los cuales han sido usados por la humanidad desde tiempos muy lejanos como los pigmentos utilizados en las pinturas rupestres de las cuevas de Altamira, en la Península Ibérica. Estos

\footnotetext{
(•) Trabajo recibido el día 23 de abril de 1997.

(*) Grupo de Corrosión y Protección. Universidad de Antioquía. A.A. 1226. Medellín (Colombia).
}

compuestos presentan una variada gama de colores que va desde el mineral prácticamente blanco (akaganeíta), hasta el negro intenso (magnetita), pasando por diversas tonalidades naranjas (lepidocrocita), rojos (hematita), marrones (goethita) y verdes (wustita). Se presentan como óxidos, hidróxidos u oxihidróxidos con o sin agua de hidratación y en estado ferroso o férrico, con propiedades físicas también bastante variadas, como aislantes, semiconductores y conductores; antiferromagnéticas, paramagnéticas o ferrimagnéticas; todo ello asociado a las diferentes formas de cristalización, que incluyen el amorfismo de la limonita y el hexagonal desordenado de la ferroxihíta, como también el ordenamiento ortorrómbico de goethita y lepidocrocita y el cúbico de espinela invertida de la magnetita y de la maghemita.

Con el transcurso de los años, y principalmente en las últimos decenios, se han visto multiplicadas sus aplicaciones, y los usos potenciales son numerosos. Esta situación ha impulsado el desarrollo de diversas vías de síntesis que permiten obtener productos controlados, a la medida, a partir de otros óxidos, de precursores oxálicos, por hidrólisis de sales de hierro, por descomposición de quelatos, etc. (1 y 2). La mayoría de las veces se sigue la vía 
de la hidrólisis (en especial, para obtener hematita, magnetita y maghemita), aunque en los últimos años se ha incrementado la síntesis mediante la técnica sol-gel (3-5). Esta permite obtener productos de gran pureza y homogeneidad en la composición, forma, tamaño y distribución de las partículas, características esenciales en las nuevas y exigentes aplicaciones tecnológicas.

A continuación, se presentan los resultados de una vasta búsqueda orientada a señalar la importancia científica y tecnológica de los compuestos químicos más relevantes: hematita, magnetita, maghemita, goethita y akaganeíta.

\section{LA HEMATITA}

La hematita, conocida también como óxido de hierro(III), especularita u oligisto, cuya fórmula química es $\alpha-\mathrm{Fe}_{2} \mathrm{O}_{3}$, tiene una masa de $70 \% \mathrm{Fe}$ y $30 \% \mathrm{O}$; es trigonal, del tipo del $\mathrm{Al}_{2} \mathrm{O}_{3}$, con parámetros de red $a_{\mathrm{o}}=5,038 \AA$ y $c_{\mathrm{o}}=12,272 \AA$. Es paramagnética y aislante eléctrica, y las partículas tienen forma de plaquetas hexagonales u octogonales, variando su color de marrón rojizo (rojo sangre) a negra. Se halla en depósitos independientes a veces de gran espesor y extensión, como mineral asociado en rocas ígneas, como inclusión en muchos minerales, en forma de producto de sublimación de lavas o como resultado de metamorfismo de contacto, y por alteración de siderita o magnetita. Por hidratación se transforma en limonita (6).

El uso de la hematita como pigmento en pinturas y barnices se encuentra muy extendido, debido a su buena resistencia a la acción de ácidos y bases. Es un buen protector para interiores, exteriores y piezas metálicas. Resiste la acción del calor y los rayos ultravioleta y tiene la ventaja de los bajos costos en la obtención de la materia prima y de su procesamiento (7-9). Para obtener diferentes gamas de color se mezcla con sílice, alúmina y óxido de calcio (10). Los pigmentos de mejor calidad tienen un elevado contenido de partículas laminares. La acción protectora está asociada al empaquetamiento de las laminillas en la película de pintura, formando capas traslapadas. Esta distribución retrasa la penetración de la humedad y de las sustancias corrosivas como los iones cloruro y sulfato.

La necesidad impuesta por las nuevas normas de prevención de la contaminación de reemplazar los pigmentos para pinturas de imprimación basados en plomo y cromatos por otros ambientalmente aceptables (11 y 12), ha acrecentado el interés por la utilización de pigmentos que actúan de "barreras" inertes, y el desarrollo de hematita sintética en forma de laminillas (hojuelas) ha sido un paso significativo en este sentido. Igualmente, se han desarrollado pinturas de alto contenido en sólidos con tales pig- mentos, los cuales, al igual que en las anteriores, aparte de su función como barrera inerte, reducen la aparición de ciertos defectos que son comúnmente observados en las pinturas de alto contenido en sólidos (10). El descubrimiento del proceso para obtener óxidos de hierro laminares sintéticos ha brindado nuevas oportunidades en el campo de las pinturas anticorrosivas. En comparación con la hematita convencional, los óxidos sintéticos laminares poseen características de barrera muy superiores. Se obtienen partículas con espesores menores de $15 \mu \mathrm{m}$ y se pueden emplear conjuntamente con pigmentos químicos activos. Además, poseen mayor capacidad de dispersión, una textura lisa y resistencia a la corrosión más elevada. En combinación con pigmentos a base de fosfato de zinc actúan de forma sinérgica aumentando las propiedades anticorrosivas del recubrimiento. El contenido de fosfato de zinc (y posiblemente el de otros pigmentos activos) se puede disminuir, lo cual significa una reducción de costos manteniendo un alto nivel de protección (7 y 8 ).

En el caśo específico de pinturas para mantenimiento y repintado de puentes, las que tienen base uretánica y están pigmentadas con óxidos de hierro laminares o micáceos muestran un comportamiento mejor que las de base epóxica y, además, su uso presenta algunas ventajas importantes como: pocas restricciones en cuanto al punto de rocío o humedad, inmersión al cabo de pocos minutos, posible aplicación en condiciones de niebla densa, aplicación rápida, habilidad para curar a temperaturas tan bajas como $15^{\circ} \mathrm{C}$, y niveles aceptables de compuestos orgánicos volátiles (13).

La hematita también se usa ampliamente en cosmética (9 y 14), donde se prefiere en la forma sintética, ya que así se evita la presencia de trazas de arsénico que podrían producir daños en personas y animales. Además, se utiliza como pigmento para caucho, papel, linóleo, cerámicas y baldosas. La hematita natural de mejor calidad y la hematita sintética se utilizan para pulir vidrio, metales preciosos y diamantes (15 y 16). Este y otros óxidos de hierro son los principales colorantes utilizados en la fabricación de vidrios (17). Igualmente, tiene uso como catalizador en diferentes procesos químicos; entre otros, se utiliza en la licuefacción de carbones bituminosos (18 y 19), en la deshidrogenación del etilbenceno (20), para la oxidación de alquilpiridinas (21) y en la descomposición de peróxido de hidrógeno (22), habiéndose encontrado que las condiciones en las que se obtiene la hematita determinan los parámetros de la subestructura y la composición de sus fases, lo que se relaciona con su acción catalítica.

A este compuesto se le atribuye un buen comportamiento como adsorbente. Por ejemplo, en investigaciones realizadas con el fin de estudiar la relación entre el enriquecimiento del oro laterítico y 
la formación de oro coloidal en medios ricos en óxidos de hierro, se realizaron adsorciones del metal en hematita sintética, observándose gran afinidad entre las partículas. Aparentemente, cualquier oro coloidal que esté en solución en un suelo determinado puede inmovilizarse fácilmente por óxidos férricos, siempre y cuando no estén presentes ciertas especies inhibidoras tales como los citratos (23). Turner y col. (24) evaluaron la adsorción de sulfatos en hematitas naturales y sintéticas, encontrando que a menor $\mathrm{pH}$ es mayor su capacidad de adsorción, y concluyendo que el proceso de adsorción es irreversible. La hematita se utiliza, además, como adsorbente de sustancias orgánicas que incluyen la poliacrilamida y el p-hidroxilbenzoato ( 25 y 26 ).

La capacidad adsorbente de la hematita se aprovecha para la fabricación de sensores. Se ha investigado su uso como sensor de flúor en mezclas gaseosas. La detección se basa en la modificación de las características semiconductoras de la hematita, debido a la adsorción del flúor, hecho que se produce a temperaturas superiores a $280{ }^{\circ} \mathrm{C}(27)$. Por su parte, Clarke y col. (28) estudiaron la adsorción de vapor de agua en hematita y otros óxidos de hierro, con miras a su posible utilización como sensores de gases, habiéndo obtenido resultados bastante aceptables. Además, se ha utilizado un material cerámico a base de hematita como detector de humedad, que presenta muy buena respuesta en el intervalo comprendido entre 0 y $95 \%$ de humedad relativa, con una elevada reproducibilidad de los resultados (29).

En el campo de la biotecnología, la hematita se ha utilizado para el tratamiento de aguas residuales. Se ha encontrado que una mezcla pulverulenta con goethita, agregada en cantidades que varían entre 25 y $50 \mathrm{mg} \cdot \mathrm{l}^{-1}$, logra intensificar la oxidación biológica en las aguas residuales y mejora las propiedades de sedimentación (30). Por otra parte, se han probado en animales tres suplementos alimenticios preparados con hematita, concluyendo que no presentan efectos cancerígenos (31).

Los imanes permanentes elaborados con ferritas hexagonales tipo $\mathrm{M}$ (materiales con fórmula química $\mathrm{MO} \cdot 6 \mathrm{Fe}_{2} \mathrm{O}_{3}$, donde $\mathrm{M}=\mathrm{Ba}, \mathrm{Pb}^{2+}$ o Sr), son los de mayor utilización industrial en nuestros días. Estas hexaferritas se obtienen, entre otras vías, mediante procedimientos cerámicos en los que se emplea polvo de hematita procedente de óxidos minerales y residuos de la industria siderúrgica o de procedimientos de síntesis (32).

La hematita mezclada con $\mathrm{Bi}_{2} \mathrm{O}_{3}$ se utiliza en la elaboración de cristales semiconductores (33 y 34). También se elaboran vidrios semiconductores al mezclarla con $\mathrm{MnO}$ y $\mathrm{P}_{2} \mathrm{O}_{5}$, o con $\mathrm{Nb}_{2} \mathrm{O}_{5}, \mathrm{P}_{2} \mathrm{O}_{5}$ y $\mathrm{LiO}_{2}$ (35 y 36). Además, se han desarrollado métodos para obtener compuestos poliméricos magnéticos con nanopartículas de hematita y maghemita, que se pueden utilizar en dispositivos para interferencia electromagnética y absorción de microondas (37).

Se ha evaluado la adición de hematita a los vidrios de pentóxido de vanadio y su efecto en las propiedades electroquímicas al usar el vidrio como cátodo en baterías recargables de litio, encontrándose que los coeficientes de difusión química son de 1 a 2 órdenes de magnitud mayores que los de $\mathrm{V}_{2} \mathrm{O}_{5}$ puro (38). Mediante el proceso sol-gel es posible formar una película delgada de hematita dopada con litio $\left(\mathrm{Li}_{\mathrm{x}} \mathrm{Fe}_{2-\mathrm{x}} \mathrm{O}_{3}\right)$, la cual se aplica en electrodos para uso en electroquímica (39).

\section{LA MAGNETITA}

La magnetita, de fórmula $\mathrm{Fe}_{3} \mathrm{O}_{4}, \mathrm{Fe}^{\mathrm{II}} \mathrm{O} \cdot \mathrm{Fe}_{2}{ }^{\mathrm{III}} \mathrm{O}_{3}$ ó $\mathrm{Fe}^{\mathrm{II}} \mathrm{Fe}_{2}{ }^{\mathrm{III}} \mathrm{O}_{4}$, se conoce como tetróxido de trihierro u óxido ferroso férrico y su color es negro. De fórmula general $\mathrm{AB}_{2} \mathrm{O}_{4}$ del grupo de la espinela $\left(\mathrm{MgO} \cdot \mathrm{Al}_{2} \mathrm{O}_{3}\right)$. Es cúbica, con parámetro $a_{0}=$ $8,3963 \AA$ A , ferrimagnética y semiconductora. El oxígeno forma la red cúbica de caras centradas, deja 32 espacios octaédricos y 64 tetraédricos; los octaédricos están ocupados por $\mathrm{Fe}^{2+}$ y $\mathrm{Fe}^{3+}$, y los tetraédricos por $\mathrm{Fe}^{3+}$. El hierro supone el $72,4 \%$ y el oxígeno el $27,6 \%$ en masa. A temperatura elevada puede cambiar ligeramente debido a que la red puede aceptar un exceso de iones trivalentes. Los iones ferrosos y férricos de las posiciones octaédricas comparten los electrones de valencia, lo que permite que sea un compuesto frecuentemente no estequiométrico y de elevada conductividad eléctrica. Se encuentra diseminada como mineral asociado a la mayoría de las rocas ígneas. Comúnmente está asociada a rocas metamórficas cristalinas formadas al abrigo del aire. Se encuentra como una placa fina o dendrita entre placas de mica, y es uno de los constituyentes de las arenas de los ríos, lagos y mares. Se altera pasando a limonita o hematita, teniendo como intermediaria a la maghemita, debido a la semejanza en la estructura cristalina (6).

Este óxido es ampliamente utilizado como pigmento para pinturas, linóleo y en la industria textil (13). Además, existen estudios que demuestran que la incorporación de óxidos de hierro en la fabricación de vidrios con borosilicato de sodio tiene efectos favorables, pues la adición de magnetita incrementa notablemente la resistencia al agua, debido a que se forma una mezcla $\mathrm{Fe}-\mathrm{Si}-\mathrm{O}$ que incrementa las propiedades protectoras de las capas superficiales (40). Se utiliza como colorante en la producción de vidrios opacos y semiopacos, en los cuales produce las tonalidades verde, azul claro y negro (41).

Por sus características abrasivas, es utilizada como compuesto para pulir (13), mientras que por sus propiedades magnéticas se emplea en la 
fabricación de imanes permanentes (41). Además, se utiliza como partículas ultrafinas en algunos dispositivos para grabaciones magnéticas y almacenamiento de datos. Para obtener partículas con características morfológicas y magnéticas apropiadas para grabación de alta densidad, se mezclan magnetita y maghemita obtenidas a partir de precursores oxálicos y el producto se dopa con cobalto y boro (42).

La magnetita, junto con la hematita, son los óxidos de hierro más utilizados como catalizadores, siendo considerada como un excelente catalizador en la descomposición del peróxido de hidrógeno (21 y 43). Igualmente, se ha estudiado su comportamiento en la adsorción de agua con resultados aceptables, por lo que se ha buscado su aplicación en sensores de gases (27).

En el campo de la biotecnología, se han preparado sintéticamente partículas de magnetita con un diámetro promedio comprendido entre 10 y $15 \mathrm{~nm}$, que se utilizan como ayuda para la inmovilización enzimática (44). Además, se ha utilizado para la inmovilización de tirosinasa, un compuesto que se emplea en el tratamiento de aguas residuales (45).

Por medio de procesos biomiméticos se pueden obtener fibras y barbas (whiskers) cerámicas de magnetita que se emplean para la elaboración de materiales compuestos que tienen aplicaciones médicas (biomateriales) e industriales (por ejemplo, en dispositivos para hornos de microondas). El producto formado mediante estos procesos tiene propiedades únicas debido a su estructura fibrosa (46).

También en el campo de la medicina existen algunos trabajos sobre la utilización de óxidos de hierro como agentes que mejoran el contraste en las imágenes de órganos humanos obtenidas por resonancia magnética nuclear (NMR). La sustancia que contienen los agentes contrastantes se inyecta a los pacientes antes del examen, sin ningún efecto aparente para el organismo (47-49). Al parecer, por recelo de los laboratorios fabricantes, generalmente no se especifica qué óxidos de hierro se incluyen en la formulación. Sin embargo, se sabe que Bulte y cols. (50) realizaron estudios con óxidos (magnetita) y oxihidróxidos (ferrihidrita, akaganeíta), con el propósito de comprender mejor su efecto en las imágenes obtenidas por este método. Por otro lado, se realizaron ensayos en animales con un suplemento de hierro que contenía magnetita, concluyéndose que no tenía efectos cancerígenos sobre los mismos (30).

\section{LA MAGHEMITA}

La maghemita es $\gamma-\mathrm{Fe}_{2} \mathrm{O}_{3}$, de color marrón, formada por partículas octogonales (6), de carácter semiconductor a aislante y ferrimagnética, por lo que también se la conoce como hematita magnética. Es cúbica, de parámetro $a_{0}=8,322 \AA$, con estructura de espinela. Poco abundante en la naturaleza, se puede obtener por oxidación de la magnetita o por deshidratación de la lepidocrocita.

El dióxido de cromo fue introducido en el decenio de 1960 como el primer material utilizable para grabaciones de alta densidad. Posteriormente, se estudió la aplicación de los óxidos de hierro para el mismo propósito. En la bibliografía se encuentran muchos trabajos en los que se estudian las características de la maghemita utilizada en dispositivos para grabación magnética: cintas de audio y de vídeo, así como discos duros y blandos de ordenadores (2 y 51-55). Las partículas de maghemita utilizadas son de grano acicular (50) y presentan una resistencia, frente a distintos medios corrosivos, superior a la de otros materiales utilizados en grabaciones magnéticas (51). En las grabaciones de alta frecuencia, la porosidad y las condiciones de preparación del óxido son factores importantes para mejorar las características de grabación (53). Los disquetes tiénen una capa magnética que contiene partículas de maghemita, y su vida útil depende de la fricción a la que esté sometida dicha capa (54).

Algunos investigadores han estudiado recientemente las propiedades de la maghemita modificada con cobalto (50 y 56-60), y con aluminio (2), a pesar de que el dióxido de cromo posee unas características semejantes y a veces superiores como medio de grabación magnética (55 y 57). Para estos estudios se ha recurrido a la deposición en fase vapor de películas delgadas de maghemita con cobalto, a partir de hematita (58), y a la obtención de maghemita modificada con cobalto, a partir de precursores oxálicos (41 y 61).

Es posible la utilización de placas delgadas de maghemita y $\mathrm{Fe}_{2} \mathrm{CoO}_{4}$, como fluidos magnéticos, en imágenes activas que se producen por medio de rayos láser (62).

La maghemita exhibe un buen comportamiento en la adsorción de vapor de agua. Por este motivo, también se busca su aplicación en la fabricación de sensores de gases (27).

Recientemente, Nguyen y col. (26) prepararon un compuesto polimérico magnético que contiene nanopartículas de maghemita, que se puede utilizar en dispositivos para interferencia electromagnética y absorción de microondas.

\section{LA GOETHITA}

La goethita, de fórmula $\alpha-\mathrm{FeOOH}, \mathrm{Fe}_{2} \mathrm{O}_{3} \cdot \mathrm{H}_{2} \mathrm{O}$ o $\mathrm{HFeO}_{2}$, es óxido férrico hidratado u óxido de hierro e hidrógeno, de estructura ortorrómbica, isomorfa de la diáspora $\left(\mathrm{HAlO}_{2}\right)$, con parámetros de 
red $a_{0}=4,64 \AA, b_{0}=10,0 \AA, c_{0}=3,03 \AA$. Es paramagnética y aislante eléctrica. Su composición, es de: $89,9 \% \mathrm{Fe}_{2} \mathrm{O}_{3}$ y $10,1 \% \mathrm{H}_{2} \mathrm{O}$ ó $62,9 \% \mathrm{Fe}, 27 \%$ $\mathrm{O}, 10,1 \% \mathrm{H}_{2} \mathrm{O}$. Constituida por partículas aciculares de color marrón amarillo a marrón oscuro. Normalmente, está formada en condiciones oxidantes, como producto de meteorización de siderita, pirita, magnetita y glauconita, por acción del agua, del dióxido de carbono, de los ácidos orgánicos y del oxígeno (6).

Es uno de los óxidos de hierro más usados en estudios de laboratorio. Sirve como sistema modelo para una gran variedad de investigaciones, en parte, porque su química superficial y su morfología están bien caracterizadas, y en parte también, porque es el óxido de hierro más común en la naturaleza. Por esa razón, muchos estudios se encaminan a la producción de goethitas con características morfológicas diferentes o con un reemplazo parcial del hierro por otros iones metálicos que pueden modificar sus propiedades (1).

La goethita se utiliza como adsorbente, aplicándola sobre fibras de carbón activado, colectoras de gases como $\mathrm{NO}, \mathrm{SO}_{2}$ y $\mathrm{NH}_{3}$, mostrando una acción bastante efectiva sobre todo en el último caso (63). $\mathrm{Al}$ doparla con cobre incrementa notablemente la capacidad de adsorción de NO, debido posiblemente a cambios en los defectos de red (64). Su capacidad adsorbente también ha sido demostrada en los trabajos de Enzweiler y col. (22) sobre el enriquecimiento del oro laterítico y la formación de oro coloidal, lo mismo que por Turner y col. (23) al estudiar la adsorción del azufre, confirmado posteriormente por Zhang y col. (65). También ha sido utilizada como adsorbente del p-hidroxibenzoato y de los monoésteres fosfatados ( 25 y 66 ).

La goethita se puede utilizar como pigmento o colorante, sobre todo la obtenida de forma sintética (67); también se usa para purificar el agua y como contraveneno del arsénico (15). La mezcla con hematita, agregada en cantidades que varían entre 25 y $50 \mathrm{mg} \cdot \mathrm{L}^{-1}$, logra intensificar la oxidación biológica en las aguas residuales y mejora las propiedades de sedimentación (29). Para la separación de metales pesados y tóxicos se puede emplear goethita, que coprecipita con ellos. Luego, se puede aplicar lixiviación bacteriana. Francis y col. (68) describen un método para la separación de cadmio, cromo, níquel, plomo y zinc, coprecipitados con goethita por medio de un microbio anaerobio. Otros investigadores utilizaron goethita para la precipitación de selenio, uranio, plomo y cromo, afirmando que la elevada reactividad de los oxihidróxidos de hierro se debe a que los grupos hidroxilos forman unos espacios entre ellos que permiten la acomodación de los metales pesados (69). Otro estudio sobre la adsorción de $\mathrm{Cr}^{3+}, \mathrm{Cd}^{2+}$ y $\mathrm{Pb}^{2+}$, con diferentes condiciones de fuerza iónica y de valores de $\mathrm{pH}$, concluye que ésta es fuertemente dependiente del $\mathrm{pH}(70)$.

El oxihidróxido se emplea como material de partida en la producción de ferritas hexagonales de tipo $\mathrm{M}$ por métodos de síntesis hidrotérmica, las cuales se utilizan ampliamente en imanes permanentes y en dispositivos para grabaciones magnéticas y magnetoópticas (31 y 71). En estos casos, se puede emplear el producto obtenido a partir de aguas de decapado (72).

Hacley y col. (73) sintetizaron y caracterizaron membranas cerámicas obtenidas a partir de goethita, que se pueden emplear como capa intermedia de soporte en sistemas cerámicos multicapas para catálisis. Por otro lado, se han realizado pruebas de un medicamento que sirve como suplemento de hierro, elaborado a partir de la goethita. Las pruebas con animales muestran que no tiene efectos canceríge$\operatorname{nos}(30)$.

\section{LA AKAGANEÍTA}

La akaganeíta, cuya fórmula es $\beta-\mathrm{FeOOH}$, es un óxido férrico hidratado, tetragonal, probablemente isomorfa del $\alpha-\mathrm{MnO}_{2}$, de parámetros $a_{0}=10,535 \AA$ y $c_{0}=3,030 \AA$ A Paramagnética y aislante eléctrica; constituida por partículas aciculares de color que va del marrón pálido al blanco. Se forma en presencia de haluros, principalmente $\mathrm{Cl}^{-}$y F . Tiene muy poca estabilidad, de manera que es bastante difícil encontrarla en la naturaleza (6).

Por poseer una estructura tubular, la akaganeíta se puede utilizar como catalizador en la síntesis de sustancias orgánicas (por ej., en polipéptidos). Su morfología permite la captación de moléculas orgánicas alargadas, lo que facilitaría la formación de péptidos a partir de aminoácidos (74-76). Según Pradel y cols. (77), los compuestos de hierro obtenidos como subproductos en la separación de hierro, desde lixiviados ácidos de la industria del zinc electrolítico, ofrecen una elevada actividad catálica en el hidroprocesamiento del carbón. El compuesto utilizado industrialmente por ellos se compone principalmente de akaganeíta.

La akaganeíta es un buen sustrato en cromatografía e intercambio iónico. Sin embargo, su aplicación en estos casos está restringida a sistemas líquidos, ya que su estructura es extremadamente sensible a la pérdida de agua (75). En medicina, se ha estudiado su utilización en sustancias que se inyectan a pacientes para obtener imágenes de resonancia magnética del organismo con un mejor contraste (49). 


\section{CONCLUSIONES}

Los óxidos de hierro naturales se han utilizado ampliamente a lo largo de la historia de la humanidad. El descubrimiento de métodos para su síntesis ha permitido extender su campo de aplicación a materiales y procesos que requieren una gran calidad y pureza, lo que les ha permitido convertirse en agentes del desarrollo de los denominados nuevos materiales, suponiéndose que aún queda mucho por descubrirles como materiales sumamente útiles al hombre.

\section{REFERENCIAS}

(1) Schwertmann, U. y Cornell, R.M. Iron oxides in the laboratory. Ed. VCH. Weinheim (RFA), 1991: 1-18

(2) DA Costa, G.M. Mössbauer spectroscopy and X-ray diffraction studies of maghemite $\{\gamma$-Fe1-yAly) $2 \mathrm{O} 3\}$ with $0.0 \leq y \leq 0.66$. Tesis doctoral. Univ. de Gante. Gante (Bélgica), 1996: 1, 103 y 104

(3) Montalvo, L., Bermudez, O. y Rodriguez, J.E. Metal. Cien. Mater. UPTC (Tunja), 4 (5), 1994: 9-13.

(4) Jansen, M. y Guenter, E. Chem. Mater., 7, 1995: 2.110 2.114.

(5) Zenitagoya, Y., Davalos, J., Peña, V. y Estrada, W. Fifth Latin American Conf. on Applications of The Mössbauer Effect. Univ. Nacional Mayor de San Marcos. Cuzco (Perú), 1996: 59.

(6) Arroyave, C. El papel del $\mathrm{NO}_{x}$ en la corrosión atmosférica del acero. Tesis doctoral. Univ. Complutense. Madrid, 1995: 61-64.

(7) CARTER, E. Anti-Corros., 33, (10), 1986: 12-14.

(8) Carter, E. J. Oil Colour Chem. Ass., 71 (5), 1988: 132133.

(9) CARTer, E. Pigment Resin Tech., Dec. 1988: 4-7.

(10) Palacio, G.L. y Restrepo, M.O. Síntesis de acetato de cobre y óxido de hierro. Univ. de Antioquia. Medellín (Colombia), 1986: 21-28.

(11) Kamani, P. Proc. ACS Division of Polymeric Mater. Sci. and Eng. Whasington DC (EE.UU.), 1990: 1.018.

(12) Del Amo, B., Romagnoli, R. y Vetere, V.F. $5^{\circ}$ Encuentro Iberoamericano de Corrosión y Protección. Univ. de La Laguna. Tenerife (España). 1995: 275-277.

(13) Anon. Better Roads, 65 (11), 1995: 23-24.

(14) Clark, G.L. y Hawley, G. The encyclopedia of chemistry. Ed. Reinhold Publ. Nueva York (EE.UU.), 1957: 273.

(15) Hurlbut, C.S. y Klein, C. Manual de Mineralogía de Dana. $3^{a}$ ed. Reverté. Barcelona (España), 1988: 287, 290,297 y 306.

(16) KIRK, R.E. y OthMER, D.F. Enciclopedia de Tecnología Química. Tomo IX. Ed. UTEHA. México, 1962: 327-329

(17) Vasilev, S.K., Orlov, D.L. y Chesnokov, A.F. Glass Ceram., 46 (1-2), 1989: 56-58.

(18) Cebolla, V.L., Diack, M., Oberson, M., Bacaud, R., Cagniant, D. y Nickel-Pepin-Donat, B. Fuel Process. Technol., 28 (2), 1991: 183-201.

(19) Yokoyama S., Yamamoto, M., Yoshida, R., Maekawa. Y. y Kotanigawa, T. Fuel, 70 (2), 1991: 163-168.
(20) Stepanov, E.G., Dvoretski, N.V., Sudzilovskaya, T.N. y Kotelnikov, G.R. Kinet. Catal., 31 (4), 1991: 823-827.

(21) Suvorov, B.V., Belova, N.A., Kan, I.I. y RaKhimova, M.A. Kinet. Catal.-Engl. Tr., 31 (2), 1990: 428-432.

(22) Mucka, V. y Mızıк, P. Radiat. Phys. Chem., 38 (3), 1991: 291-294.

(23) Enzweiler, J. y Joekes, I. J. Geochem. Explor., 40 (1-3), 1991: 133-142.

(24) Turner, L.J. y Kramer, J.R. Water Air Soil Pollut., 63 (1-2), 1992: 23-32.

(25) Lee, L.T. y Somasundaran, P. J. Colloid Interface Sci., 42 (2), 1991: 470-479.

(26) KunG, K.H. y McBride, M.B. Clays Clay Min., 37 (4), 1989: 333-340.

(27) Vasiliev, A.A., Godovsky, D., Buturlin, A. y GabuZyan, T. Sensor Actuator B-Chem., B14 (1-3), 1993: 705-707.

(28) Clarke, N.S. y Hall, P.G. Langmuir, V.7 (4), 1991: 672-677.

(29) Pelino, M., Colella, C., Cantalini, C., Faccio, M., FERri, G. y D'Amico, A. Sensor Actuator B-Chem., B7 (1-3), 1992: 464-469.

(30) Verkhovtseva, N.V., Lebedeva, N.A. y Kanevskaya, L.I. Sov. J. Water Chem. Technol., 11 (6), 1989: 98-100.

(31) Steinhoff, D., Mohr, U. y Hanhemann, S. Exp. Pathol., 43 (3-4), 1991: 189-194.

(32) Dufour, J., Negro, C., Latorre, R., Lopez-Mateos, F. y Formoso, A. Rev. Metal. Madrid, 31 (2), 1995: 111-119.

(33) Chaudhuri, B.K., Chaudhuri, K. y Som, K. J. Phys. Chem. Solids, 50 (11), 1989: 1.149-1.155.

(34) Chaudhuri, B.K., Chaudhuri, K. y Som, K. J. Phys. Chem. Solids, 50 (11), 1989: 1.137-1.147.

(35) De Araujo, D.B., de Figueiredo, R.S. y de Araujo, M.A.B. Fifth Latin American Conf. on Applications of The Mössbauer Effect. Univ. Nacional Mayor de San Marcos. Cuzco (Perú), 1996: 22.

(36) De Araujo, M.A.B., de Magalhaes Abreu, J.A., DE Paiva, J.A.C., DE Figueiredo, R.S. y Sombra, A.S.B. Fifth Latin American Conf. on Applications of The Mössbauer Effect. Univ. Nacional Mayor de San Marcos. Cuzco (Perú). 1996: 23.

(37) Nguyen, My. T. y Diaz, A.F. Advan. Mater., 6 (11), 1994: 858-860.

(38) MACHIDA M., FUCHIDA, R. y MinAmi, T. J. Electrochem. Soc., 137 (4), 1990: 1.315-1.316.

(39) Campet, G., Wen, S.J., Han, S.D., Shastry, M.C.R., Portier, J., Guizard, C., Cot, L., Xu, Y. y Salardenne, J.. Mater. Sci. Eng. B-Solid-State M., B18 (2), 1993: 201-208.

(40) Kuznetsov, A.I., Kruchinin, D., Fedotova, L.M. y Kruchinin, D. Sov. J. Glass Phys. Chem., 14 (4), 1989: 337-341.

(41) McColm, I.J. Dictionary of ceramic science and engineering. 2a. ed. Ed. Plenum Press. Nueva York (EE.UU.), 1994: 57, 197 y 229.

(42) Tailhades, Ph., Sarda, Ch., Bonino, Ch., Mollard, P. y Rousset, A. J. Mag. Magn. Mat., 89 (1-2), 1990: 33-37.

(43) Fubini, B., Mollo, L. y Giamello, E. Free. Radic. Res., 23 (6), 1995: 593-614.

(44) Kobayashi, H. y Matsunaga, T. J. Colloid Interface Sci., 141 (2), 1991: 505-511.

(45) Wada, S., Ichikawa, H. y Tatsumi, K. Biotechnol. Bioeng., 45 (4), 1995: 304-309. 
(46) Lawton, C.W. y Shields, Ch. Biomolecular Materials. Materials Research Society Symposium Proc. Pittsburgh (EE.UU.), 1993: 107-114.

(47) WeISSLEDER, R. Radiology, 193 (3), 1994: S75-S77.

(48) Rogers, J., Lewis, J. y Josephson, L. Mag. Reson. Imaging., 12 (4), 1994: 631-639.

(49) Chambon, C., Clement, O., Le-Blanche, A., Schouman-Claeys, E. y FriJa, G. Mag. Reson. Imaging., 11 (4), 1993: 509-519.

(50) Bulte, J.M., Vymazal, J., Brooks, R.A., Pierpaoli, C. y Frank, J.A. J. Mag. Reson. Imaging., 3 (4), 1993:

(51) Sharrock, M.P. IEEE Trans. Mag., 25 (6), 1989: 4.3744.389.

(52) Speliotia, D.E. IEEE Trans. Mag., 26 (1), 1990: 124126.

(53) Huang, Y.Ch., Fowkes, F.M. y Lloyd, T.B. J. Adhes. Sci. Technol., 5 (1), 1991: 39-56.

(54) Corradi, A.R., Green, W.B., Price, T.G., Bottoni, G., Candolfo, D., Cecchetti, A., Masoli, F. y Molesini, L. IEEE Trans. Mag., 26 (1), 1990: 237-240.

(55) Tanaka, K., Ueda, S. y Enoki, Y.. Surf. Coat. Technol., 44, (1-3), 1990: 790-798.

(56) Auweter, H., Feser, R., Jakusch, H., Muller, M.W., Muller, N., Schwab, E. y Veitch, R.J. IEEE Trans. Mag., 26 (1), 1990: 66-68.

(57) Corradi, A.R., Dinitto, C.A., Price, T.G. y Green, W.B. IEEE Trans. Mag., 26 (5), 1990: 1.888-1.890.

(58) Bottoni, G., Candolfo, D., Cecchetti, A., Corradi, A.R. y Masoli, F. IEEE Trans. Mag., 26 (5), 1990: $1.885-1.887$

(59) Dhara, S., Rastogi, A.C. y Das, B.K. Bull. Mat. Sci., 17 (4), 1994: 367-387.

(60) Borges, J.F.M., Timm, C.A. y DA Costa Jr., M.I. Fifth Latin American Conf. on Applications of The Mössbauer
Effect. Univ. Nacional Mayor de San Marcos. Cuzco (Perú), 1996: 133.

(61) Gougeon, M., Leroy, E., Tailhades, Ph., Mollard, P. y Rousset, A. IEEE Trans. Mag., 26 (1), 1990: 57-59.

(62) Gergiland, P.Y., Le Jeune, B., Cariou, J. y Lotrian, J. J. Mag. Magn. Mat., 122 (1-3), 1993: 406-410.

(63) Kaneko, K. y Shindo, N. Carbon, 27 (6), 1989: 815-820.

(64) Kaneko, K., Wang, Z., Suzuki, T., Ozeki, S., Kosugi, N. y Kuroda, H. J. Colloid Interface Sci., 142 (2), 1991: 489-496.

(65) Zhang, P.Ch. y Sparks, D.L. Soil Sci. Soc. Amer. J., 54 (5), 1990: 1.266-1.273.

(66) Ognalaga, M., Frossard, E. y Thomas, F. Soil Sci. Soc. Amer J., 58 (2), 1994: 332-337.

(67) Kraus, E.H. Mineralogía. Ed. Nueva York, 1965: 307.

(68) Francis, A.J. y Dodge, C.J. Environ. Sci. Technol., 24 (3), 1990: 373-378.

(69) Manceau, A., Charlet, L., Boisset, M.C., Didier, B. y SPAdini, L. Appl. Clay Sci., 7 (1-3), 1992: 201-223.

(70) Jinadasa, K.B.P.N., DissanayaKe, C.B. y WeERasooriya, S.V.R. Int. J. Environ. Stud. A \& B., 48 (1), 1995: 7-16.

(71) Wang, M.L., Shin, Z.W. y Lin, Ch. J. Cryst. Growth, 130: (1-2), 1993: 153-161.

(72) O'Connor, D.L., Dudokovic, M.P. y RAMACHANDRAN, P.A. Ind. Eng. Chem. Res., 31 (11), 1992: 2.516-2.524.

(73) Hacley, V.A. y Anderson, M.A. J. Membrane Sci., 70 (1), 1992: 41-51

(74) Holm, N.G., Dowler, M.J. y Wadsten, T. Geochim. Cosmochim. Acta, 47, 1983: 1.465-1.470.

(75) Holm, N.G. Origins Life, 14 ,1984: 343-350.

(76) Holm, N.G. Origins Life, 15, 1985: 131-139.

(77) Pradel, J., Castillo, S., Traverse, J.P., Grezes-Besset, R. y Darcy, M. Ind. Eng. Chem. Res., 32 (9), 1993: 1.8011.804 . 